\title{
Efficacy and safety of tianeptine in the treatment of depressive disorders in comparison with fluoxetine
}

\author{
H. Lôo ${ }^{1}$, J. Saiz-Ruiz², J. A. Costa e Silva³ ${ }^{3}$ M. Ansseau4, R. Herrington ${ }^{5}$, A. Vaz-Serra6, H. Dilling7 and \\ S. De Risio ${ }^{8}$ \\ ${ }^{1}$ Service Hospitalo-universitaire de Santé Mentale et de Thérapeutique. Sainte-Anne Hospital, Paris Cedex \\ 14, France \\ ${ }^{2}$ Ramon y Cajal Hospital, Department of Psychiatry, Madrid, Spain \\ ${ }^{3}$ University Hospital, Faculty of Medicine, Rio de Janeiro, Brazil \\ ${ }^{4}$ University Hospital, Neuropsychiatric Clinic, Liège, Belgium \\ ${ }^{5}$ Gartnavel Royal Hospital, Glasgow, Scotland, UK \\ ${ }^{6}$ University Hospital, Department of Psychiatry, Coimbra, Portugal \\ ${ }^{7}$ University Hospital, Clinic of Psychiatry, Lu beck, Germany \\ ${ }^{8}$ Catholic University del Sacro Cuore, Institute of Psychiatry and Psychology, Rome, Italy
}

KEYWORDS: depressive disorders, fluoxetine, tianeptine

\section{ABSTRACT}

Depression is treated by a great variety of antidepressant treatments. SSRIs (such as fluoxetine) are well known: it is, however, sure that further progress is needed and the search for antidepressants with other mechanisms of action (such as tianeptine) or different efficacy is still of interest. A multinational study compared tianeptine with fluoxetine in 387 patients with Depressive Episode, or Recurrent Depressive Disorder, or Bipolar Affective Disorder (ICD-10), in a double-blind parallel group design. They were treated for six weeks. At inclusion, no significant difference between groups was shown. Final MADRS scores were 15.7 and 15.8 with tianeptine and fluoxetine, respectively (ITT population) $(p=0.944)$. MADRS responders were $58 \%$ and $56 \%$ with tianeptine and fluoxetine, respectively $(p=0.710)$. No statistical difference was observed for the other efficacy parameters. Thirty-six withdrawals occurred in each group, without any difference for the reasons of discontinuation. There was no major difference between groups for the other safety parameters. In this study, both tianeptine and fluoxetine exhibited a good efficacy and safety. 


\section{Introduction}

Tianeptine is an antidepressant which differs from the conventional antidepressants by its unique property of increasing 5HT uptake in the synaptic cleft. In pharmacological studies, its antidepressive activity has been shown using the classical screening tests of antagonism of reserpine-like compounds, rat behavioural despair (Porsolt's test) and aggressive behaviour induced by isolation in mice (Poignant, 1981; Mocaër, 1988). It has been demonstrated that tianeptine was active in the bulbectomized rat model of depression (Kelly and Leonard, 1990) and in stress models of depression such as immobilisation stress (Whitton et al., 1991) and inescapable shocks in the learned helplessness paradigm (Thiébot et al., 1992).

Neurochemistry studies have shown that tianeptine increases serotonin uptake in rat brain (Mennini et al., 1987), and rat (Kato and Weitsch, 1988) and human platelets (Renaud et al., 1988; Chamba et al., 1991) after acute and chronic administration.

Tianeptine is currently administered three times a day (12.5 mg X 3/day). Its antidepressant efficacy is comparable to those of imipramine and amitriptyline (Ostaptzeff, 1981; Weiss et al., 1981; Lôo et al., 1988; Guelfi et al., 1989; Guelfi, 1992; Invernizzi et al., 1994). Until recently, most of comparative studies with tianeptine were performed versus usual tricyclics or placebo.

However, a comparative study versus fluoxetine has already been performed (Alby et al., 1993). This study was carried out in 206 depressed patients: it showed no difference between tianeptine and fluoxetine in respect of their antidepressive efficacy. Some limitations in data interpretation made it necessary to conduct a second trial, which is presented in this paper. Further comments on the first study will be made in the discussion section.

Tianeptine has been shown to be effective in patients suffering from depression alone or associated with anxiety or alcoholism. Its safety has been confirmed in more than 3000 patients treated in open and controlled studies for one month to one year (Delalleau et al., 1988; Chapuy et al., 1991; Guelfi et al., 1992; Lôo et al., 1990, 1992). The comparison studies showed a better safety profile in comparison to trycyclic antidepressants: lower rate of adverse events, fewer atropinic or cardiovascular side-effects, fewer sedative properties.

Adverse effects are infrequent and mostly mild: digestive disorders (such as abdominal pain, nausea, vomiting, constipation, dry mouth), insomnia, drowsiness, headache and tachycardia.

Fluoxetine is a selective serotonin reuptake inhibitor which was demonstrated to be effective in the treatment of major depressive episode (Montgomery, 1995). Its adverse effects are mostly mild and rare: tremors, insomnia, headache, drowsiness, digestive disorders (nausea, diarrhoea, dry mouth), rarely cutaneous disorders and cardiovascular disorders (bradycardia).

The aim of the current study was to compare the efficacy and safety of tianeptine with those of fluoxetine, an SSRI antidepressant, in the treatment of depressive disorders. 


\section{METHODS}

\section{STUDY DESIGN}

This study was a multicentre, international, phase III double-blind controlled trial. All patients were included between October 1995 and November 1996. Ten countries participated in the trial: Austria, Belgium, Brazil, France, Germany, Italy, the Netherlands, Portugal, Spain and United Kingdom. In each country, the study protocol was submitted to the relevant Ethics Committee for approval: the study began in each centre only after receiving the written approval of the Ethics Committee on which it depended.

In each country, the training of the investigators for the rating of the scales used in this trial was made by the use of video tapes of depressed patients.

Patients were treated in a parallel group design either with tianeptine (12.5 mg t.i.d., at $8 \mathrm{am}$, noon and $8 \mathrm{pm}$ ) or with fluoxetine (20 mg once a day at $8 \mathrm{am}$, then placebo at noon and $8 \mathrm{pm}$ ) for 42 days, after a one-week run-in placebo period. The aim of the placebo pre-inclusion week was to exclude rapid placebo-responders.

\section{INCLUSION CRITERIA}

Men and women, aged from 18 to 65 years, in- or out- patients were selected if they met the following inclusion criteria: Depressive episode, Recurrent depressive disorder or Bipolar affective disorder (depressed) according to the ICD-10 classification (moderate or severe episode, with or without somatic syndrome, without psychotic symptoms); having a MADRS (Montgomery and Asberg, 1979) score $\geq 25$ points, and requiring an antidepressant treatment.

All patients freely gave their written informed consent to participate in the study.

\section{EXCLUSION CRITERIA}

The patients who were not allowed to participate in the study had other types of depression (especially dysthymia and double depression), a severe risk of suicide, an acute or chronic psychosis or had failed to respond to two antidepressants for the current depressive episode. Other exclusion criteria were previous history of drug abuse or dependence, severe somatic diseases in evolution, and current treatment with barbiturates, buspirone, thymoregulators, antiepileptic drugs. Diazepam was forbidden because of potential interaction with fluoxetine: lorazepam and alprazolam were not allowed as some publications have shown that they may possess some antidepressive properties.

\section{CONCOMITANT TREATMENTS}

No concomitant psychotropic medication was allowed, but if the patient had used a benzodiazepine (except those mentioned under Exclusion Criteria) for at least one month before the trial, he/she was to continue taking it. If a patient without a benzodiazepine needed an 
anxiolytic treatment during the trial, he could only receive clorazepate (maximum dosage: 30 $\mathrm{mg} /$ day) or oxazepam (maximum dosage: $60 \mathrm{mg} /$ day). The prescription of somatic treatments was permitted provided they did not interfere with depression.

\section{ASSESSMENTS}

Patients were assessed at selection (D-7), at the inclusion (D1) and then during the study at D7, D14, D28 and D42.

At the selection visit (D-7), a complete psychiatric interview (history of depression and of the present depressive episode, check for inclusion and exclusion criteria) and a complete physical examination were performed. Laboratory parameters with haematology (RBC, WBC, haemoglobin, differential blood count, platelets, haematocrit) and biochemistry (glucose, creatinine, ALAT, ASAT, ÎGT, alkaline phosphatase, total bilirubin) were also measured.

At D1, patients were definitively included if they still fulfilled the inclusion criteria, if the decrease in the MADRS score was lower than $30 \%$, and this score remained higher than 25 points.

At each visit, D7, D14, D28 and D42, the severity of the depressive symptomatology was assessed with the MADRS and CGI (Clinical Global Impression) scale. In order to document the evolution of the somatic complaints of the patients and to search for any adverse events, the AMDP5 scale (Bobon, 1981) was used at each evaluation.

At the last visit (D42), a medical examination was performed and laboratory parameters were also measured.

Compliance was measured at each visit by the count of the number of capsules brought back by the patients.

\section{STATISTICAL ANALYSIS}

The main efficacy criterion was the final MADRS global score. The secondary efficacy criteria were a decrease of 50\% in MADRS global score (responder patients), and the CGI scores.

The safety was evaluated with emergent adverse events, somatic complaints at each visit and with vital signs and biological parameters at D-7 and D42.

Student's $t$ test for independent samples was performed for quantitative variables and a Fisher's exact test for qualitative variables. Differences between the two groups were tested with a Student's $t$ test. The change over the time comparison was performed with a two-way analysis of variance (group X time) with repeated measures. Delay of responses were studied in terms of survival curves according to the Kaplan-Meier method. Survival curves were compared using the log-rank test. The main analysis was performed on the intention-to-treat (ITT) population using the last observation carried forward method and secondary analyses on the per-protocol (PP) population. 
The minimum number of patients for the study was 300; it was calculated on the basis of a difference of 4 points in final MADRS scores between treatment groups, with $\alpha$ risk $=5 \%, \beta$ risk $=10 \%$, and a standard deviation of 10 .

All analyses were performed using SAS V6.09 software. For all statistical tests, the type I error threshold was set at 5\%.

\section{Results}

\section{DESCRIPTION OF THE POPULATION}

Three hundred and eighty-seven patients were randomized (191 to the tianeptine group and 196 to the fluoxetine group). The ITT population was madeup of 381 patients (187 in the tianeptine group and 194 in the fluoxetine group); six patients had baseline measurements and no evaluation on treatment, and were therefore excluded from the intention-to-treat analysis. The PP population (patients who completed the study duration without major deviation from the protocol) represented 266 patients (131 in the tianeptine group and 135 in the fluoxetine group).

One hundred and ninety-seven patients (51\%) were included in France, 61 patients (16\%) in Spain, 45 patients (12\%) in Brazil and the remaining 84 patients in Belgium, United Kingdom, Austria, Portugal, Germany, Italy and the Netherlands. No group X country effect was observed at inclusion or at the end of the treatment.

\section{DEMOGRAPHIC AND CLINICAL CHARACTERISTICS OF THE PATIENTS}

At inclusion, no significant differences appeared between the two groups.

Table 1 presents the demographic and clinical characteristics of the included patients. The mean age was $41.8 \pm 11.0$ years in the tianeptine group and $41.0 \pm 11.4$ in the fluoxetine group. One hundred and thirty-five patients (72\%) and 147 patients (76\%) respectively were women. Thirty-two patients in each group were hospitalized at inclusion.

In the tianeptine group, 79 (42\%) patients presented a depressive episode, 106 (57\%) a recurrent depressive disorder and $2(1 \%)$ a bipolar disorder. In the fluoxetine group, 73 (38\%) presented a depressive episode, $121(62 \%)$ a recurrent depressive episode and none a bipolar disorder. These differences were not significant $(p=0.374)$.

The severity of the depression was moderate for 128 (68\%) patients in the tianeptine group and 126 (65\%) in the fluoxetine group; it was severe for 59 (32\%) patients in the tianeptine group and $68(35 \%)$ in the fluoxetine group. Eighty-five (45\%) patients in the tianeptine group and 92 $(47 \%)$ in the fluoxetine group resented a somatic syndrome $(p=0.778)$. The 179 patients of the randomized population who presented a somatic syndrome had the following characteristics: they were more frequently hospitalised than those who had no somatic syndrome $(22.4 \% \mathrm{vs}$ $11.5 \%$ ) and were more severely depressed (MADRS global score $33.3 \pm 5.2$ vs $30.9 \pm 4.5$ ). Sixtyeight patients with severe depression (53\%) had no somatic syndrome. 
It was the first depressive episode for $74(40 \%)$ patients in the tianeptine group and for 66 (34\%) patients in the fluoxetine group. The mean duration of the current episode was $6.8 \pm 14.4$ months in the tianeptine group and 7.2 \pm 16.4 months in the fluoxetine group $(p=0.835)$.

Forty-four of 110 patients (39\%) in the tianeptine group and 47/126 (37\%) patients in the fluoxetine group had a history of three or more depressive episodes. The mean duration since the first depressive episode was $124.6 \pm 111$ months in the tianeptine group and $114.0 \pm 109$ months in the fluoxetine group ( $p=0.455)$. Sixty-three (34\%) patients in the tianeptine group and $56(29 \%)$ in the fluoxetine group had a family history of depression.

The initial MADRS global score was $32.12 \pm 4.80$ points in the tianeptine group and of $31.93 \pm$ 5.13 in the fluoxetine group ( $p=0.702$ ). The first item of the CGI (severity of illness) was $4.64 \pm$ 0.69 in the tianeptine group and $4.69 \pm 0.79$ in the fluoxetine group $(p=0.476)$.

Table 1. Demographic characteristics of the patients (ITT population)

\begin{tabular}{cccc}
\hline & $\begin{array}{c}\text { Tianeptine } \\
\mathrm{n}=187 \\
\text { mean } \pm \text { S.D. }\end{array}$ & $\begin{array}{c}\text { Fluoxetine } \\
\mathrm{n}=194 \\
\text { mean } \pm \text { S.D. }\end{array}$ & $p$ \\
Mex & $52(28 \%)$ & $47(24 \%)$ & 0.496 \\
Women & $135(72 \%)$ & $147(76 \%)$ & 0.465 \\
Age (years) & $41.8 \pm 11.0$ & $41.0 \pm 11.4$ & 0.829 \\
Weight (kg) & $68.9 \pm 15.9$ & $68.5 \pm 17.2$ & \\
Height (cm) & $165.1 \pm 8.5$ & $164.2 \pm 7.7$ & 0.374 \\
Depressive episode & $79(42 \%)$ & $73(38 \%)$ & 0.835 \\
Recurrent depression & $106(57 \%)$ & $121(62 \%)$ & 0.455 \\
Bipolar disorder & $2(1 \%)$ & 0 & \\
Duration of the current & $6.8 \pm 14.4$ & $7.2 \pm 16.4$ & \\
episode (months) & $124.6 \pm 110.7$ & $114.0 \pm 108.5$ & \\
Duration since the first & & \\
episode (months) & $75(39 \%)$ & $66(34 \%)$ & \\
First episode & $43 / 110(39 \%)$ & $47 / 126(37 \%)$ & \\
Three previous episodes or & & \\
more & $63(34 \%)$ & $56(29 \%)$ & \\
Family history of depression & & &
\end{tabular}

\section{EFFICACY}

At the end of the treatment, in the ITT population the global score on the MADRS was $15.69 \pm$ 10.85 in the tianeptine group and $15.77 \pm 11.19$ in the fluoxetine group ( $p=0.944)$. In the PP population the difference was not significant either $(p=0.967)$. The first item of the CGI (severity of illness) was $2.79 \pm 1.44$ in the tianeptine group and $2.75 \pm 1.45$ in the fluoxetine 
group ( $p=0.821$ ). The second item of the CGI (global improvement) was $2.21 \pm 1.33$ in the tianeptine group and $2.22 \pm 1.25$ in the fluoxetine group $(p=0.984)$. The third item of the CGI (efficacy index) was $2.83 \pm 1.24$ in the tianeptine group and $2.79 \pm 1.26$ in the fluoxetine group $(p=0.719)$.

Considering the time course of the MADRS scores in the PP population, the scores decreased significantly at each visit $(p=0.01)$ and for both groups the difference between D1 and D42 was highly significant $(p=0.001)$, but the difference was not significant between the two treatment groups ( $p=0.418$ ) (Table 2). Item 10 of the MADRS (suicidal thoughts) did not differ between the two groups in the ITT population at $\mathrm{D} 42(p=0.709)$.

The delay of response to treatment (log-rank test on survival curves) was not significantly different between the two groups ( $p=0.722)$. At D42, in the ITT population, 109 (58\%) patients in the tianeptine group and $109(56 \%)$ patients in the fluoxetine group were responders $(p=$ 0.710): in the PP population 89 (68\%) patients in the tianeptine group and 86 (64\%) patients in the fluoxetine group ( $p=0.550$ ) were considered as responders to the treatment according to the MADRS score. The differences were not significant, but the percentage of responders was slightly higher in the tianeptine group. The analysis in survival curves did not show any difference between the two groups.

The investigators considered the efficacy as good or excellent for $63 \%$ of the patients in each group (Figure 1). From the patients' point of view, $59 \%$ in the tianeptine group and $63.5 \%$ in the fluoxetine group considered that the treatment had good efficacy (Figure 2).

Table 2. Evolution of MADRS score (PP population)

\begin{tabular}{lccccc}
\hline $\begin{array}{l}\text { PP } \\
\text { population }\end{array}$ & $\begin{array}{c}\text { Tianeptine } n=131 \\
\text { mean } \pm \text { S.D. }\end{array}$ & $\begin{array}{c}\text { Fluoxetine } n=134 \\
\text { mean } \pm \text { S.D. }\end{array}$ & $\begin{array}{c}\text { Time X group } \\
\text { interaction }\end{array}$ & $\begin{array}{c}\text { Group effect } \\
P\end{array}$ & $\begin{array}{c}\text { Time effect } \\
P\end{array}$ \\
\hline D1 & $31.8 \pm 4.60$ & $30.99 \pm 3.84$ & & & \\
D7 & $26.1 \pm 6.78$ & $26.40 \pm 5.77$ & & & \\
D14 & $20.95 \pm 7.58$ & $21.11 \pm 7.75$ & 0.418 & 0.646 & $<0.001$ \\
D28 & $16.36 \pm 7.42$ & $15.42 \pm 8.07$ & & & \\
D42 & $12.85 \pm 8.06$ & $12.91 \pm 8.12$ & & & \\
\hline
\end{tabular}

\section{SAFETY}

No laboratory parameters of vital signs differed significantly throughout the trial in any group or between the two groups.

During the trial, $40-45 \%$ of patients received a benzodiazepine treatment. This percentage was not different between groups and did not change during the study.

At inclusion, the most common somatic complaints recorded using the AMDP5 scale were interruption of sleep (73\%), difficulty falling asleep (67\%), shortened duration of sleep (64\%), 
decreased appetite (59\%) and decreased libido (58\%). Most somatic complaints disappeared in both treatment groups: this improvement is likely to be secondary to the improvement in the depression of the patients. However, more patients complained of nausea, gastric discomfort, diarrhoea, palpitations, backache, chills and tremor with fluoxetine than with tianeptine. Conversely, more patients complained of shortened sleep, excessive thirst, cardiac pain and paraesthesia with tianeptine than with fluoxetine.

Withdrawals were observed in 72 patients (36 patients in each group). The reasons were adverse events (15 and 16 patients for tianeptine and fluoxetine groups, respectively), inefficacy (12 and 8 patients for tianeptine and fluoxetine groups, respectively), non-medical reasons (8 and 9 patients for tianeptine and fluoxetine groups, respectively), patients lost to follow-up (one patient in each group) and major deviation for 2 patients in the fluoxetine group. The serious adverse events with tianeptine were suicide attempts (3 cases), worsening of depression (3 cases), arthralgia (1 case), malaise with dizziness ( 1 case), weight increase (1 case); furthermore one patient became pregnant. One of the suicide attempts was successful. The serious adverse events with fluoxetine were suicide attempts (3 cases), active plans for suicide ( 1 case), agitation ( 1 case), fall (1 case), eczema (1 case); furthermore one patient became pregnant. One of the patients who discontinued fluoxetine because of an adverse event fulfilled the criteria suggested by Sternbach (1991) for serotonin syndrome.

The investigator considered the safety as satisfactory for $88 \%$ of the patients in the tianeptine group and for $86 \%$ in the fluoxetine group (Figure 3). From the patients' point of view, $86 \%$ and $84 \%$ respectively judged the safety as satisfactory.

One hundred and eighty-six patients in the tianeptine group and 190 patients in the fluoxetine group (out of a total of 376 patients, 97.2\%) had good compliance.

\section{Efficacy}

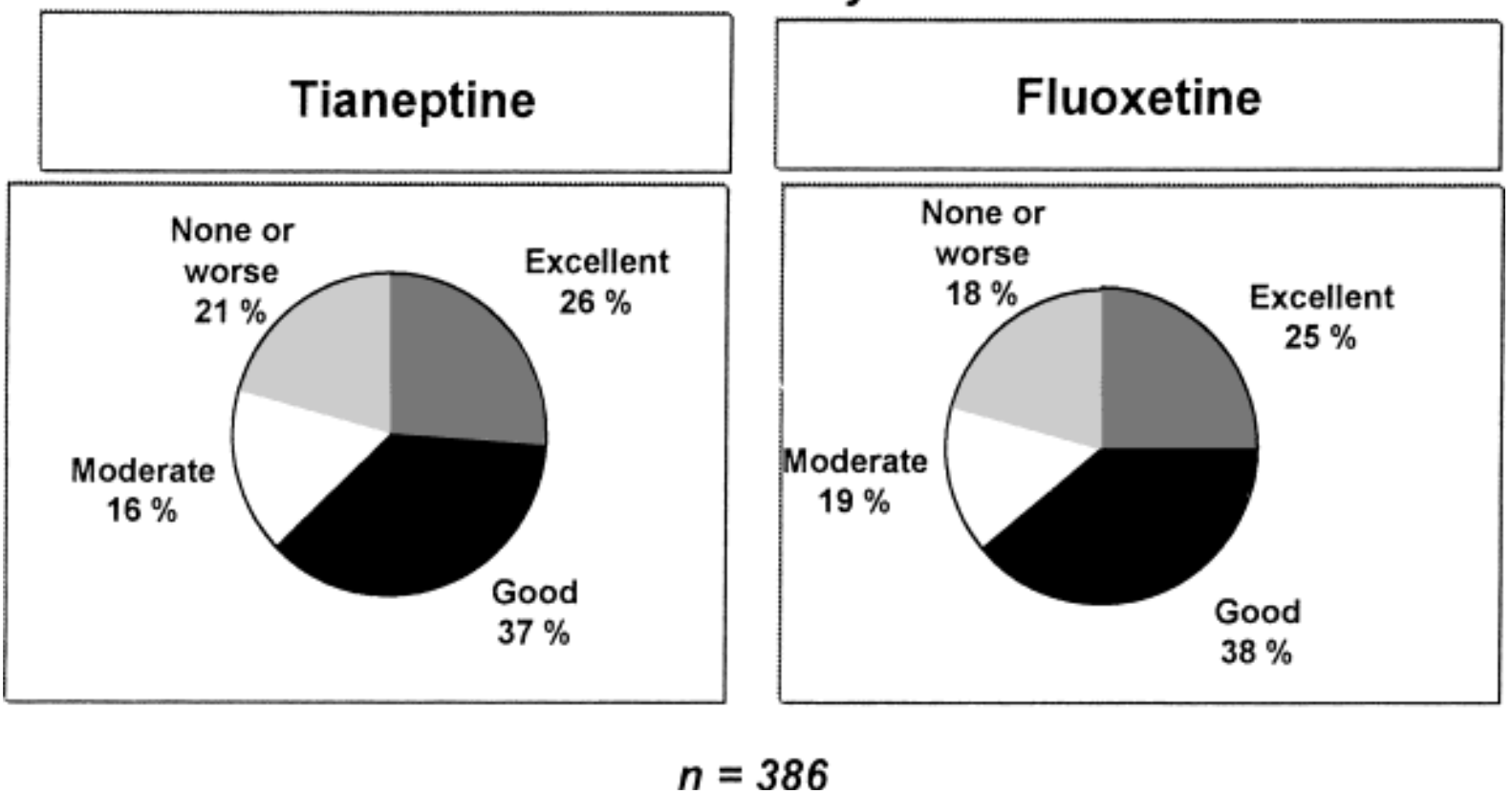

Figure 1. Global evaluation by the investigators 


\section{Efficacy}
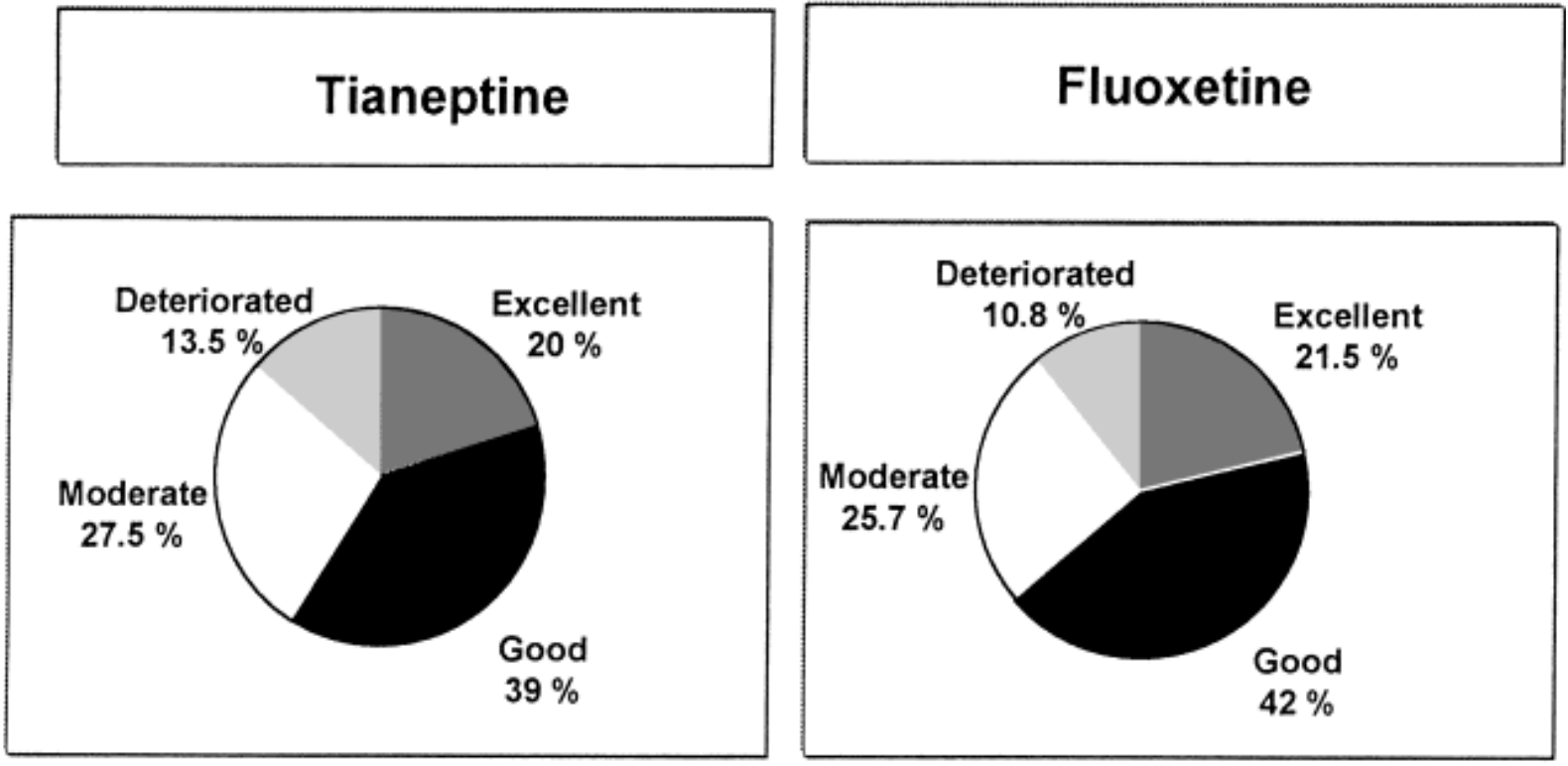

$$
n=365
$$

Figure 2. Global impression of the patients

\section{Adverse events}
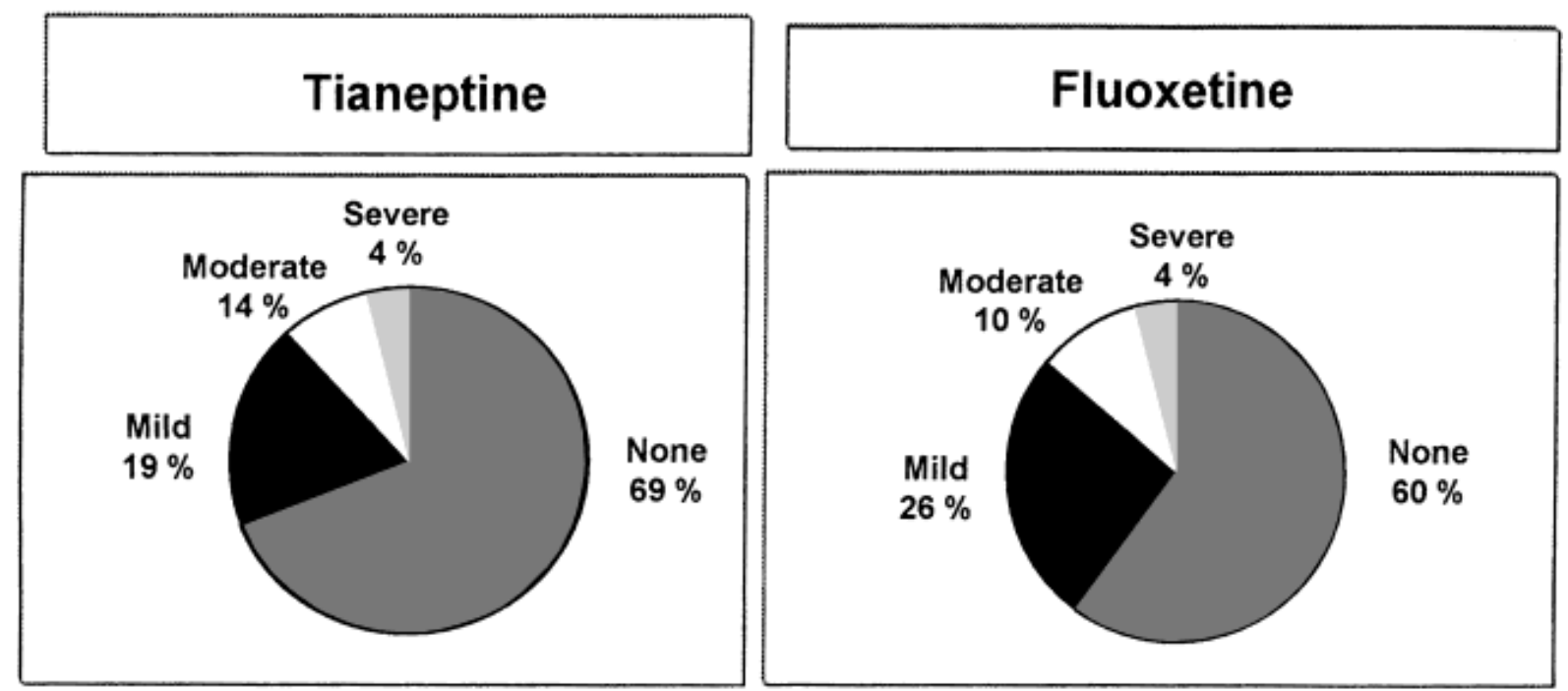

$$
n=386
$$

Figure 3. Global evaluation of the safety by the investigators 


\section{DISCUSSION}

As mentioned in the Introduction, Alby et al. (1993) have already carried out a double-blind study comparing tianeptine and fluoxetine. This study was performed in 206 patients, presenting either a major depressive episode or dysthymia (DSM III-R), and somatic complaints (assessed by a check-list of somatic complaints). The combination of patients with different diagnoses in the same trial is always questionable and the analysis of separate subgroups of patients usually leads to a decrease in the power of the statistical analysis. Furthermore, the assessment tools which were used for measuring the efficacy could not permit a comparison of the results with those of other trials in the literature.

The present study aimed to compare the efficacy and safety of tianeptine and fluoxetine in the treatment of depressive disorders diagnosed using ICD-10 criteria. The choice of ICD-10 for the inclusion of the patients was made because of the availability of this diagnostic system in the different languages of the different countries involved in this trial. Interestingly, while the ICD10 considers that most of the severe depressions should have a somatic syndrome, only $47 \%$ of these severely depressed patients had this syndrome in this trial; additional data from other studies may permit the significance of this diagnosis to be evaluated in the future. Some authors, Hiller et al. (1994), have already discussed the issue of the reliability of new diagnostic systems (ICD-10, DSM III and DSM III-R). Their conclusion was that the ICD- 10 diagnosis of 'depressive disorder' is in close relationship with that of 'major depression' of DSM III/ DSM III-R. Such a congruence had been criticised by Philipp et al. (1991) who suggested that ICD-10 did not bring a new major advance when compared with other diagnostic systems. Gross and Huber (1996) made a critical review of both ICD-10 and DSM IV systems and concluded that some aspects of these classifications of depressive or mood disorders may be questionable. Hiller et al. (1994) made an evaluation of the ICD-10 subcategories of depressive episode and recurrent depressive disorder and of the different severity categories (based on ICD-10) to see if both diagnoses could be clearly distinguished and this was not case for moderate and severe depressive syndromes. Some authors, Gross and Huber (1996), suggested that the somatic syndrome, as defined in ICD10 diagnostic system, may lack consistency, some items (early awakening, worsening in the morning, loss of interest, psychomotor retardation) being more valid than the others. However, other authors, Hamdi et al. (1997), evaluated the severity of depression using the HDRS in depressed patients with somatic syndrome versus those without this syndrome; in their study, the former were shown to be more severely depressed. This result is in agreement with those of Faravelli et al. (1996) and with our own results: the condition of the patients with somatic syndrome is more severe. Both tianeptine and fluoxetine showed their good efficacy and acceptability in the treatment of depressed patients. In fact, MADRS scores decreased significantly in both groups, even after seven days of treatment, and then continued to decrease progressively until the last evaluation at D42. It could have been interesting to have a placebo group in this study, so that the onset of action of both antidepressants can be documented by a statistically significant difference versus placebo. Forty to $45 \%$ of the patients received a benzodiazepine treatment during the study: this is in agreement with the everyday practice in some European countries; as the rates of prescription of benzodiazepines remained similar in both tianeptine and fluoxetine groups, this is not a major drawback for the conclusions of the study. Only 31 patients were withdrawn from the study because of adverse events. The 
distribution was equal in both groups, 15 and 16 patients. Twenty patients were withdrawn because of inefficacy (12 and 8 patients, respectively). This is in favour of the good efficacy and safety of these two treatments. Especially, the safety of tianeptine, already shown in the previous studies, was confirmed. Thus, according to both the investigator's judgement and the patient's point of view the efficacy was considered as good or excellent in more than $60 \%$, and the safety in more than $80 \%$, of cases.

This study has permitted the efficacy of tianeptine in treating ICD-10 depressive disorders to be demonstrated; it appeared to be as effective and safe as fluoxetine. 


\section{References}

Alby JM, Ferreri M, Cabane J, de Bodinat C, Dagens V. 1993. Efficacité de la tianeptine (STABLON ${ }^{\circledR}$ ) dans le traitement des dépressions majeures et des dysthymies, avec plaintes somatiques. Etude comparée versus fluoxétine (PROZAC ${ }^{\circledR}$ ). Ann Psychiatrie 8(2): 136-144.

Bobon DP. 1981. Le système AMDP. In Psycho Sci Hum, 2e ed. coll, Bruxelles: Mardaga.

Chamba G, Lemoine P, Flachaire E, et al. 1991. Increased serotonin platelet uptake after tianeptine administration in depressed patients. Biol Psychiatry 30: 609-617.

Chapuy P, Cuny G, Delomier Y, Galley P, Michel JP, Pareaud M, Marey C. 1991. La dépression du sujet âgé. Intérêt de la tianeptine chez 140 patients traités pendant un an. Press Med 20(37): 1844-1852.

Delalleau B, Dulcire C, Le Moine P, Kamoun A. 1988. Analysis of the side-effects of tianeptine. Clin Neuropharmacol 11 (Suppl 2): S83-S89.

Faravelli C, Servi P, Arends JA, Strik WK. 1996. Number of symptoms. Quantification, and Qualification of Depression. Compr. Psychiatry 37(5): 307-315.

Gross G, Huber G. 1996. Depressive or Mood Disorders in Operationalized Classification Systems and the Affective Psychoses (Manic-Depressive Illness. Cyclothymia) of the Traditional Psychiatry. A critical review. Neurol Psychiatry and Brain Res 4: 149-158.

Guelfi JD. 1992. Efficacy of tianeptine in comparative trials versus reference antidepressants: an overview. Br J Psychiatry 160 (Suppl 15): 72-75.

Guelfi JD, Dulcire C, Le Moine P, Tafani A. 1992. Clinical safety and efficacy of tianeptine in 1858 depressed patients treated in general practice. Neuropsychobiology 25: 140-148.

Guelfi JD, Pichot P, Dreyfus JF. 1989. Efficacy of tianeptine in anxious-depressed patients: Results of a controlled multicenter trial versus amitriptyline. Neuropsychobiology 22: 41-48.

Hamdi E, Amin Y, Abou-Saleh MT. 1997. Problems in validating endogenous depression in the Arab culture by contemporary diagnostic criteria. J Affect Disord 44: 131-143.

Hiller W, Dichtl G, Hecht H, Hundt W, Mombourg W, von Zerssen D. 1994. Evaluating the new ICD-10 categories of depressive episode and recurrent depressive disorder. J Affect Disord 31: 49-60.

Invernizzi G, Aguglia E, Bertolino A, et al. 1994. The efficacy and safety of tianeptine in the treatment of depressive disorder: results of a controlled double-blind multicentre study versus amitriptyline. Neuropsychobiology 30: 85-93.

Kato G, Weitsch AF. 1988. Neurochemical profile of tianeptine a new antidepressant drug. Clin Neuropharmacol 11 (suppl 2): S43-S50.

Kelly JP, Leonard BE. 1990. The effect of tianeptine on the olfactory bulbectomized (OB) rat model of depression. IIIrd International Congress of European Behavioural Pharmacology Meeting, Noordwigkhout (The Netherlands), 25-27 June 1990. Psychopharmacology 101 (Suppl): S28.

Lôo H, Malka R, Defrance R, et al. 1988. Tianeptine and amitriptyline: controlled double-blind trial in depressed alcoholic patients. Neuropsychobiology 19: 79-85.

Lôo H, Ganry H, et al. 1990. Acceptabilité et efficacité thérapeutique de la tianeptine chez 510 patients déprimés traités en un an. JAMA 11(hors série), 53. 
Lôo H, Ganry H, Dufòur H, et al. 1992. Long-term use of tianeptine in 380 depressed patients. Br J Psychiatry 160 (Suppl 15): 61-65.

Mennini T, Mocaer E, Garattini S. 1987. Tianeptine, a selective enhancer of serotonin uptake in rat brain. Naunyn Schmiedeberg's Arch Pharmacol 336: 478-482.

Mocaër E, Rettori MC, Kamoun A. 1988. Pharmacological antidepressive effects and tianeptine induced 5HT uptake increase. Clin Neuropharmacol 11 (Suppl 2): S32-S42.

Montgomery SA. 1995. Selective Serotonin Reuptake Inhibitors in the Acute Treatment of Depression. In Bloom FE, Kupfer DJ. (eds), Psychopharmacology-The Fourth Generation of Progress. Raven Press: New York.

Montgomery SA, Asberg M. 1979. A new depression scale designed to be sensitive to change. Br J Psychiatry 134: 382-389.

Ostaptzeff G. 1981. Etude contrôlée à double insu versus imipramine de l'efficacité de la tianeptine dans des états dépressifs non psychotiques. Biol Psychiatry 5: 597-600.

Philipp M, Maier W, Delmo CD. 1991. The concept of major depression. I. Descriptive comparison of six competing operational definitions including ICD-10 and DSM-III-R. Eur Arch Psychiatry Clin Neurosci 240: 258-265.

Poignant JC. 1981. Etude pharmacologique d'un nouvel antidépresseur: la tianeptine. Biol Psychiatry 5: 573-578.

Renaud B, Mocaër E, Weitsch AF, Kato G, Mennini T, Garattini S. 1988. Stimulation of serotonin uptake induced by a new antidepressant. ECNP Constituent meeting. Brussels (Belgium), May 1987, Pharmacopsychiatry 21: 66 .

Sternbach H. 1991. The Serotonin Syndrome. Am J Psychiatry 148(6): 705-713.

Thiébot MH, Martin P, Puech AJ. 1992. Animal behavioural studies in the evaluation of antidepressant drugs. Br J Psychiatry 160(Suppl 15): 44-50.

Weiss C, Gorceix A, Kindynis S, Dimitriu M. 1981. Etude contrôlée à double insu versus nomifensine de l'activité et du délai d'action d'un nouvel antidépresseur la tianeptine. Biol Psychiatry 5: 593-596.

Whitton PS, Sarna GS, Curzon G. 1991. Effects of tianeptine on stress-induced behavioural deficits and 5HT dependent behaviour. Psychopharmacology 104: 81-85. 